\title{
Shortest path algorithm of a network via spherical fuzzy digraphs
}

DOI:10.36909/jer.ICMMM.12393

\author{
Parimala Mani ${ }^{*}$ Ibtesam Alshammari ${ }^{* *}$ and Halimah Alshehri ${ }^{* * *}$
}

*Department of Mathematics, Bannari Amman Institute of Technology, Sathyamangalam638401, India

Email: rishwanthpari@gmail.com

** Department of Mathematics, Faculty of Science, University of Hafr Al Batin-31991, Saudi Arabia.

Email: iealshamri@hotmail.com, iealshamri@uhb.edu.sa; Corresponding Author.

**** Department of Computer Science and Engineering, Faculty of Applied Science and Community Service, King Saud University, Riyadh, Saudi Arabia.

Email: haalshehri@ksu.edu.sa

\section{ABSTRACT}

Many extension and generalization of fuzzy sets have been introduced and studied in the literature. Picture fuzzy set acquired more concentration in the domain of decision making, as many real time circumstances might have choices of more than one and researchers are looking for optimum choice/decision. Spherical fuzzy digraph is a generalization of intuitionistic fuzzy set and fuzzy graph. In this paper, we redefine some preliminary operations of Spherical fuzzy graph and it is referred as spherical fuzzy digraph. We discuss some arithmetic operations and relations among spherical fuzzy digraph. We further proposed a method to solve a shortest path problem using score function.

Key words: spherical fuzzy set; spherical fuzzy graphs; spherical fuzzy digraphs; score functions. 


\section{INTRODUCTION}

The short distance, in digital electronics and wireless communication, using multifunction sensors, it is transmitted. Sensors of this kind consist of the data being sensed and processed. Systems embedded, wireless networking, distributed processing, micro-electromechanical systems and wireless sensor applications are the main features of this technology. Sensors technology advancements and these innovations, the broad transformation has led a large contribution to the wireless sensor networks. In the context of both business and our everyday life, sensors support and improve work performance. The sensor network device is away from the real time situation and a large amount of data could be obtained and processed. Sense awareness is used by these sensors. The network and algorithm of the sensor must have the capability of organizing themselves.

Neighbourhood nodes are similar to one another, and for continuous sensing, the nodes are used. In contrast to other sensors, multichip sensor networks are used for less power consumption. Every node of the sensor network will provide topological information. For messaging from any source to any destination, the shortest path algorithms are used. In most cases, BA is used for a network with wide reaching secured nodes.

Intuitionistic fuzzy set theory (IFS) was developed by Atanassov et al., in 1980s which has got a tremendous applications in real life (Atanassov \& Stoeva, 1983, Atanassov, 1986 \& Atanassov, 1988), which is a generalizations of fuzzy set theory (FS) (Zadeh, 1965) and is applied in many real life (Özdemir \& Üsküdar, 2020). A new relations and graph structure called intuitionistic fuzzy relations (IFR) and intuitionistic fuzzy graph (IFG) respectively were then developed (Shannon \& Atanassov, 2006), which are the generalizations of fuzzy graphs (FG) (Rosenfeld, 1975). The different mathematical operations on intuitionistic fuzzy graphs are described by Parvathi et al. in (Parvathi \& Karunambigai, 2006, Parvathi et al.,2009 \& Parvathi \& Thamizhendhi, 2010). 
It has got numerous applications in engineering, social science information technology and medicines. Significantly in the areas of cluster analysis, control theory, expert systems, information theory, logistics, transportation, telecommunication, machine learning $(\mathrm{Xu} \&$ $\mathrm{Hu}, 2010$ ), manufacturing, material sciences, medical diagnosis, neural networks, social network, etc.

But, IFS somewhat restricts the researchers in a fixed scope, i.e., one cannot have the membership (m) as 0.7 and non-membership (n) grades as 0.6 simultaneously, as their sum $(m+n)$ outdo the unity. Understanding this, Yager introduced the novel notion of Pythagorean fuzzy set (PyFS) theory (Yager, 2013 \& Yager, 2014), which also contains the membership (m) and non-membership (n) grades but with an upgraded constraint to meet the more general condition, i.e., $\mathrm{m}^{2}+\mathrm{n}^{2} \leq 1$, which is a new class of unconventional type of fuzzy sets and has got numerous potential implementations in social and natural sciences See, (Garg 2017, Garg 2018a, Garg 2018b, Garg 2019 \& Wei et al., 2018).

IFS and PyFS theories failed to handle some real time situations, where neutrality plays a part of the situation. Each earthling usually will have different point of view, so they have more than one answer for any question like: yes, no, abstain and refusal. To break this kind of scenarios, the notion of picture fuzzy set (PFS) and spherical fuzzy set(SFS), as improved models of PyFS, IFS and FS theories, and were proposed and developed (Cuong \& Kreinovich, 2013 \& Ashraf et al., 2019).

As unreliability situations are clearly represented via Spherical fuzzy sets (Akram et al., 2020). The concept of Spherical fuzzy graph (SFG) was applied it decision making, which gave an outstanding research direction for the uncertain environmental optimization problems. The notion of SFS (Ashraf et al., 2019), SFG (Akram et al., 2020) and the shortest path algorithms (Parimala et al., 2019 \& Broumi et al., 2019) motivated us to propose this novel notion of Spherical fuzzy digraph. Also, in this paper a new technique is proposed for finding the solution of a shortest path problem in a network, in which the arcs length (edge) 
are indicated by Spherical fuzzy numbers.

The basic notations of SFS, SFGs and related operations of SFGs are presented in section 2. In section 3, a novel idea of Spherical fuzzy digraphs (SFDG) is introduced and also established some of its arithmetic operations and a score function of a SFN is defined. In Section 4, an algorithm and a numerical example (Network terminology problem) are proposed and solved using the score function defined in previous section is used to determine the the shortest distance and the shortest path from the source to the terminus (or destination) node of the network. The conclusion of this proposed work is fundamentally encapsulated and scope directions of future research in this novel concept are furnished in section 5 .

\section{Definition (Ashraf et al., 2019)}

\section{PRELIMINARIES}

Let $\mathrm{P}$ be a Spherical fuzzy set (SFS). $\mathrm{P}$ in $\mathrm{U}$ is defined by $\mathrm{P}=\left\{\zeta,\left(\mu_{\mathrm{P}}(\zeta), \eta_{\mathrm{P}}(\zeta), \nu_{\mathrm{P}}(\zeta)\right) \mid \zeta \in \mathrm{U}\right\}$, where $\mu_{\mathrm{P}}(\zeta), \eta_{\mathrm{P}}(\zeta), \nu_{\mathrm{P}}(\zeta) \in[0,1]$, denotes the positive, neutral (abstain) and negative membership degrees, respectively of the element $\zeta$ in $U$ and with the constraint $0 \leq \mu_{\mathrm{P}}^{2}(\zeta)+\eta_{\mathrm{P}}^{2}(\zeta)+v_{\mathrm{P}}^{2}(\zeta) \leq 1$. The refusal membership value is defined as $\pi_{\mathrm{P}}^{2}(\zeta)=1$ $\left[\mu_{\mathrm{P}}^{2}(\zeta)+\eta_{\mathrm{P}}{ }^{2}(\zeta)+v_{\mathrm{P}}{ }^{2}(\zeta)\right]$. For short, $\alpha=\left(\mu_{\alpha}, v_{\alpha}\right)$ is said to be a SFN, where $\mu_{\alpha}{ }^{2}+\eta_{\alpha}{ }^{2}+v_{\alpha}{ }^{2} \leq 1$, $\mu_{\alpha}, v_{\alpha} \in[0,1]$ and $\pi_{\alpha}^{2}(\zeta)=1-\left[\mu_{\alpha}^{2}+\eta_{\alpha}^{2}+v_{\alpha}^{2}\right]$.

The principal disparity with the IFS, Pythagorean fuzzy set(PyFS), picture fuzzy set(PFS) and SFS is their constraint conditions, i.e for the IFS the constraint condition is $\mu_{\alpha}+v_{\alpha} \leq 1$, for the PyFS the constraint condition is $\mu_{\alpha}{ }^{2}+v_{\alpha}{ }^{2} \leq 1$, for the PFS the constraint condition is $\mu_{\alpha}+\eta_{\alpha}+v_{\alpha} \leq 1$ and for the SFS the constraint condition is $\mu_{\alpha}{ }^{2}+\eta_{\alpha}{ }^{2}+v_{\alpha}{ }^{2} \leq 1$. Therefore, the membership degree space of SFS is greater than the membership degree space of IFS, PyFS and PFS, as shown in Figure 1. 


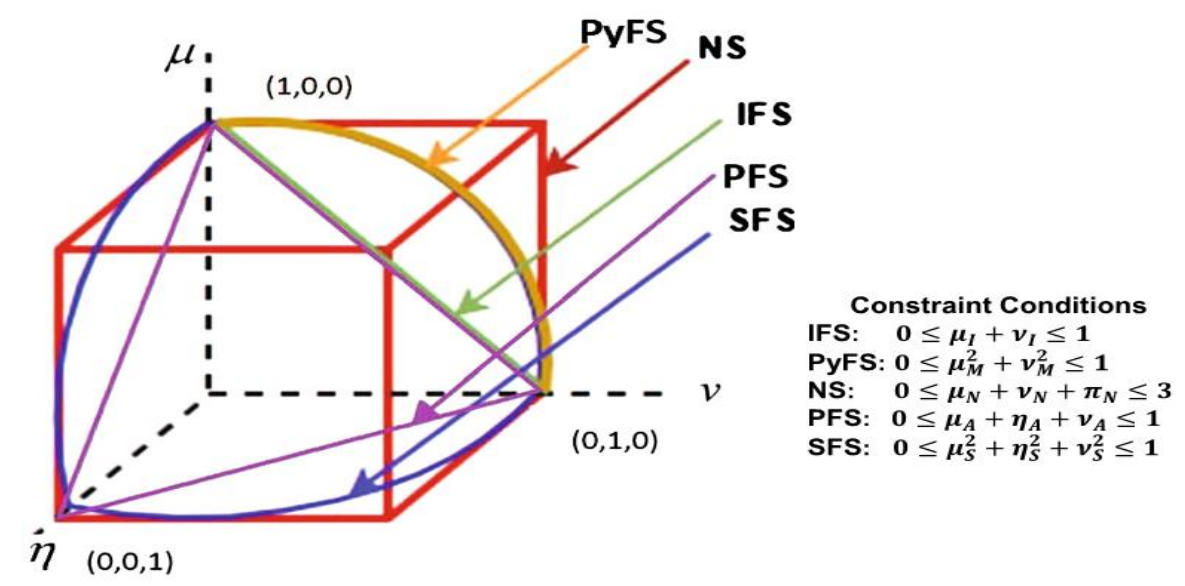

Figure 1 Space comparison between the IFSs, PyFSs, PFSs and SFSs

Definition (Akram et al., 2020)

A pair $G=(A, B)$ is called a Spherical fuzzy graph $(S F G)$ on $G^{*}$, where $G^{*}=(P, E)$ be a graph and $\mathrm{P}=\left(\mu_{\mathrm{P}}(\zeta), \eta_{\mathrm{P}}(\zeta), \nu_{\mathrm{P}}(\zeta)\right)$ is a SFS on $\mathrm{P}$ and $\mathrm{E}=\left(\mu_{\mathrm{E}}(\zeta), \eta_{\mathrm{E}}(\zeta), \nu_{\mathrm{E}}(\zeta)\right)$ is a SFS on $\mathrm{E} \subseteq$ $\mathrm{V} \times \mathrm{V} \exists, \forall \operatorname{arc} \zeta_{1} \zeta_{2} \in \mathrm{E} . \mu_{\mathrm{E}}\left(\zeta_{1}, \zeta_{2}\right) \leq \mu_{\mathrm{P}}\left(\zeta_{1}\right) \wedge \mu_{\mathrm{P}}\left(\zeta_{2}\right), \eta_{\mathrm{E}}\left(\zeta_{1}, \zeta_{2}\right) \leq \eta_{\mathrm{P}}\left(\zeta_{1}\right) \wedge \eta_{\mathrm{P}}\left(\zeta_{2}\right), v_{\mathrm{E}}\left(\zeta_{1}, \zeta_{2}\right) \leq$ $v_{\mathrm{P}}\left(\zeta_{1}\right) \vee v_{\mathrm{P}}\left(\zeta_{2}\right)$

\section{SPHERICAL FUZZY DIGRAPH}

\section{Definition}

Let $\mathrm{V}$ be a finite Spherical fuzzy non-void set $\mathrm{P}=\left(\mathrm{V}, \mu_{\mathrm{i}}, v_{\mathrm{i}}\right)$ is a SFS on $\mathrm{V}$ and $\mathrm{E}=\left(\mathrm{V} \times \mathrm{V}, \mu_{\mathrm{i}, \mathrm{j}}\right.$, $\left.\eta_{i, j}, v_{i, j}\right)$ is a Spherical fuzzy relation on $V$, then the ordered pair $G=(P, E)$ is said to be a Spherical fuzzy digraph (or) directed graph (SFDG), where $\mu_{\mathrm{i}}, \eta_{\mathrm{i}}, v_{\mathrm{i}}: \mathrm{V} \rightarrow[0,1]$, denotes the positive, neutral (abstain) and negative membership degrees, respectively for every element of the vertex $v_{i}$ in $V$ and with the constraint $0 \leq \mu_{i}^{2}\left(v_{i}\right)+\eta_{i}{ }^{2}\left(v_{i}\right)+v_{i}{ }^{2}\left(v_{i}\right) \leq 1$. And $E \subseteq V \times V$ where $\mu_{\mathrm{i}, \mathrm{j}}, \eta_{\mathrm{i}, \mathrm{j}}, v_{\mathrm{i}, \mathrm{j}}: \mathrm{V} \times \mathrm{V} \rightarrow[0,1]$, denotes the positive, abstinence and negative membership degrees, respectively for every element of the edge $e_{i j}=\left(v_{i}, v_{j}\right)$ in $V$ and with the constraint $0 \leq$ $\mu_{i, j}^{2}+v_{i, j}^{2} \leq 1 . \mu_{i, j} \leq \mu_{i}\left(v_{i}\right) \wedge \mu_{j}\left(v_{j}\right), \eta_{i, j} \leq \eta_{i}\left(v_{i}\right) \wedge \eta_{j}\left(v_{j}\right), v_{i, j} \leq v_{i}\left(v_{i}\right) \vee v_{j}\left(v_{j}\right)$.

Remark: SFDG do not have a symmetric relation on V, but a SFG has a symmetric relation on $\mathrm{V}$.

\section{Example}


Let $\mathrm{G}=(\mathrm{V}, \mathrm{E})$ be a SFDG with the vertices $\mathrm{V}=\{\mathrm{a}, \mathrm{b}, \mathrm{c}, \mathrm{d}, \mathrm{e}\}$, where the $\mathrm{SFN}$ of the vertices are $a=(0.6,0.5,0.4), b=(0.8,0.3,0.4), c=(0.6,0.3,0.2), d=(0.2,0.5,0.1), e=(0.7,0.2,0.2)$. The graph is shown in Fig.2 and the index matrix is given in the Table.1.

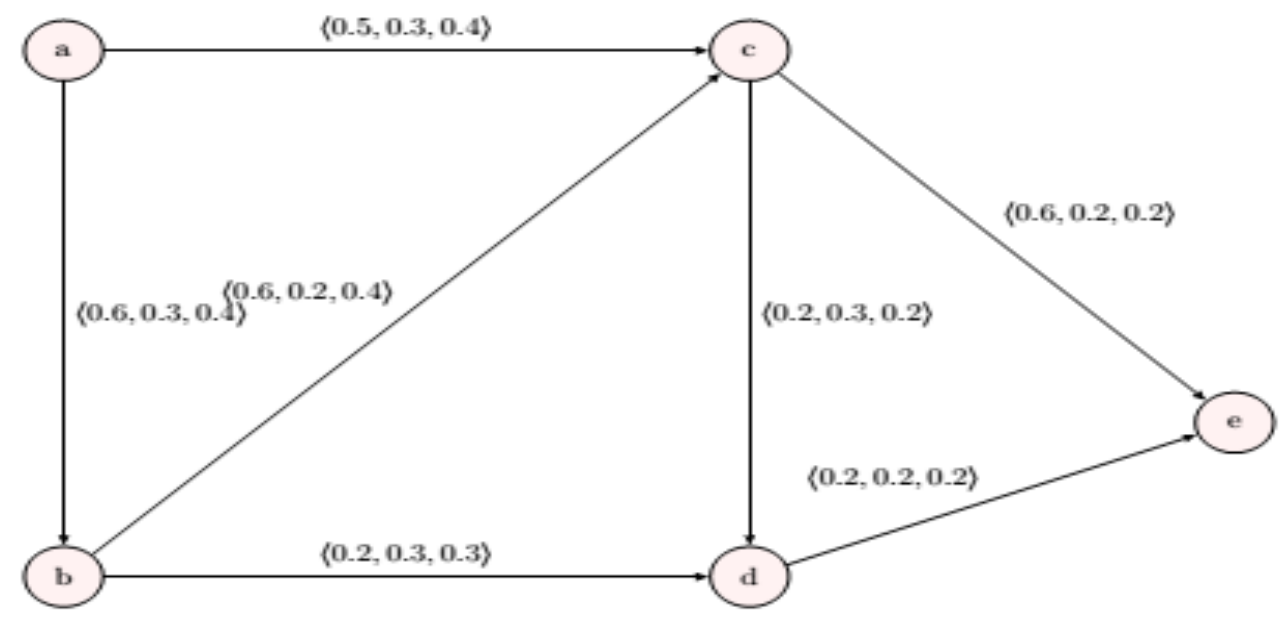

Figure 2. Spherical fuzzy digraph $\mathrm{G}$

Table 1 Index matrix of the graph $\mathrm{G}$

\begin{tabular}{|l|l|l|l|l|l|}
\hline Vertices & a & $\mathbf{b}$ & $\mathbf{c}$ & $\mathbf{d}$ & $\mathbf{e}$ \\
\hline $\mathbf{a}$ & $(0,0,1)$ & $(0.6,0.3,0.4)$ & $(0.5,0.3,0.4)$ & $(0,0,1)$ & $(0,0,1)$ \\
\hline $\mathbf{b}$ & $(0,0,1)$ & $(0,0,1)$ & $(0.6,0.2,0.4)$ & $(0.2,0.3,0.3)$ & $(0,0,1)$ \\
\hline $\mathbf{c}$ & $(0,0,1)$ & $(0,0,1)$ & $(0,0,1)$ & $(0.2,0.3,0.2)$ & $(0.6,0.2,0.2)$ \\
\hline $\mathbf{d}$ & $(0,0,1)$ & $(0,0,1)$ & $(0,0,1)$ & $(0,0,1)$ & $(0.2,0.2,0.2)$ \\
\hline e & $(0,0,1)$ & $(0,0,1)$ & $(0,0,1)$ & $(0,0,1)$ & $(0,0,1)$ \\
\hline
\end{tabular}

\section{Definition}

The operations for Spherical fuzzy numbers (SFNs) are given below for the two SFNs S1 = $(\mu 1, \eta 1, v 1)$ and $S 2=(\mu 2, \eta 2, \nu 2)$

1. $P_{1} \oplus P_{2}=\left(\sqrt{\mu_{1}^{2}+\mu_{2}^{2}-\left(\mu_{1}\right)^{2}\left(\mu_{2}\right)^{2}}, \sqrt{\eta_{1}^{2}+\eta_{2}^{2}-\left(\eta_{1}\right)^{2}\left(\eta_{2}\right)^{2}}, v_{1} v_{2}\right)$

2. $P_{1} \otimes P_{2}=\left(\mu_{1} \mu_{2}, \eta_{1} \eta_{2} \sqrt{v_{1}^{2}+v_{2}^{2}-\left(v_{1}\right)^{2}\left(v_{2}\right)^{2}}\right)$

3. $\alpha P_{1}=\left(\sqrt{1-\left(1-\mu_{1}^{2}\right)^{\alpha}}, \sqrt{1-\left(1-\eta_{1}^{2}\right)^{\alpha}}, v_{1}^{\alpha}\right)$ 
4. $P_{1}^{\alpha}=\left(\mu_{1}^{\alpha}, \eta_{1}^{\alpha}, \sqrt{1-\left(1-v_{1}^{2}\right)^{\alpha}}\right)$

\section{Definition}

The score function $\mathrm{S}(\mathrm{P})$ and the accuracy function $\mathrm{A}(\mathrm{P})$ for a SFN $\mathrm{P}=(\mu, \eta, v)$ are defined as follows

1. $S(P)=[(\mu-v) 2-(\eta-v) 2]$

2. $\mathrm{A}(\mathrm{P})=\mu 2+\eta 2+v 2$

Comparison of two SFNs $\mathrm{P} 1=(\mathrm{m} 1, \mathrm{a} 1, \mathrm{n} 1)$ and $\mathrm{P} 2=(\mathrm{m} 2, \mathrm{a} 2, \mathrm{n} 2)$ is done as follows

1. if $\mathrm{S}(\mathrm{P} 1)<\mathrm{S}(\mathrm{P} 2)$, then $\mathrm{P} 1<\mathrm{P} 2$,

2. if $\mathrm{S}(\mathrm{P} 1)=\mathrm{S}(\mathrm{P} 2)$, then

a. if $\mathrm{A}(\mathrm{P} 1)<\mathrm{A}(\mathrm{P} 2)$, then $\mathrm{P} 1<\mathrm{P} 2$,

b. if $\mathrm{A}(\mathrm{P} 1)=\mathrm{A}(\mathrm{P} 2)$, then $\mathrm{P} 1=\mathrm{P} 2$.

\section{NETWORK TERMINOLOGY AND PROPOSED ALGORITHM FOR A SPHERICAL FUZZY DIRECTED GRAPHS}

In this manuscript, the arc length of a network is contemplated as SFN, also in this section, to find the shortest path and distance in a Spherical fuzzy set network from the source to the terminus node, an algorithm is proposed. This labeling technique algorithm can be applied for finding real time problem's shortest path (SP).

Let $G=(V, E)$ be a SFDG with a finite set of nodes $V=\{1,2,3, \ldots, n\}$ and a finite set of directed $\operatorname{arcs}($ edges) $\mathrm{E}=\{1,2,3, \ldots, \mathrm{m}\}$. In this network, we insist two nodes namely, source node (1) and the terminus node (n). Here we denote the path $\mathrm{Pij}=\{$ alternating nodes and arcs from source to the terminus nodes $\}$. The existence of a minimum of one path P1i in the digraph is supposed for every $i$ in $\mathrm{V}-\{1\}$. The SFN related to the edge (i,j), with respect to the length required to pass over $(I, j)$ from i to $j$. $d(P)$ denotes the Spherical fuzzy distance along the path and is defined as $\mathrm{d}(\mathrm{P})=\Sigma \mathrm{dij}$ 


\section{Algorithm}

Step-1: Let $\mathrm{p}_{1}=(0,0,1)$ and the source node is labeled as $\mathrm{p}_{1}$

Step-2: Compute $\mathrm{p}_{\mathrm{j}}=\min \left\{\mathrm{p}_{\mathrm{i}} \oplus \mathrm{p}_{\mathrm{ij}}\right\}$, for $\mathrm{j}=1,2,3, \ldots, \mathrm{n}$

Step-3: To the unique value of $i$, there exist a minimum corresponding to it, i.e., $i=q$, then label node $\mathrm{j}$ as $\left[\mathrm{p}_{\mathrm{j}}, \mathrm{q}\right]$.

Step-4: Let the end node (n) be labeled as $\left[\mathrm{p}_{n}, 1\right]$, then the Spherical fuzzy shortest distance from source node is $\mathrm{p}_{\mathrm{n}}$.

Step-5: Reason being the label of end node is denoted as $\left[p_{n}, 1\right]$. Now, to compute the Spherical fuzzy shortest path from the source to the terminus node, examine the label of node 1. Let it be $\left[p_{n}, r\right]$, now look over the label of node $r$ and so on. This course of action is repeated till node 1 is achieved.

Step-6: Combining all the nodes identified by step-5 gives the Spherical fuzzy shortest path.

\section{NUMERICAL EXAMPLE FOR THE PROPOSED ALGORITHM}

A bike manufacture, wishes to provide a premier after sales service to the customers. If the customer has some problem in the bike, then he approaches the showroom where he purchased the bike to get recuperate the issue. The showroom mechanic of the company will direct this issue to the service center (branches) of the company in different cities. The service centers are linked with each other service centers as if the issue is big, they can approaches

the other. There are three possible case arises in this case the first one is the possibility to clear the issue by the service center (positive membership), the second one is non availability technicians or spare parts (abstinence) and the third one is not possible to clear the issue (negative membership). The proposed algorithm helps the manufacturer to find best service providing center through and the shortest path.

Table 2. Spherical fuzzy weights (SFW) of the network graph G. 


\begin{tabular}{|l|l|l|l|l|c|}
\hline S.No. & Arc name & \multicolumn{1}{|c|}{ SFW } & S.No. & Arc name & SFW \\
\hline 1. & $(1,2)$ & $(0.7,0.5,0.3)$ & 8. & $(4,7)$ & $(0.3,0.3,0.3)$ \\
\hline 2. & $(1,3)$ & $(0.6,0.2,0.4)$ & 9. & $(5,6)$ & $(0.6,0.2,0.1)$ \\
\hline 3. & $(2,3)$ & $(0.5,0.6,0.2)$ & 10. & $(5,7)$ & $(0.5,0.2,0.2)$ \\
\hline 4. & $(2,5)$ & $(0.8,0.4,0.1)$ & 11. & $(5,8)$ & $(0.6,0.3,0.4)$ \\
\hline 5. & $(3,4)$ & $(0.9,0.1,0.2)$ & 12. & $(6,8)$ & $(0.8,0.3,0.1)$ \\
\hline 6. & $(3,5)$ & $(0.7,0.5,0.4)$ & 13. & $(6,7)$ & $(0.6,0.1,0.1)$ \\
\hline 7. & $(4,5)$ & $(0.3,0.6,0.5)$ & 14. & $(7,8)$ & $(0.5,0.2,0.4)$ \\
\hline
\end{tabular}

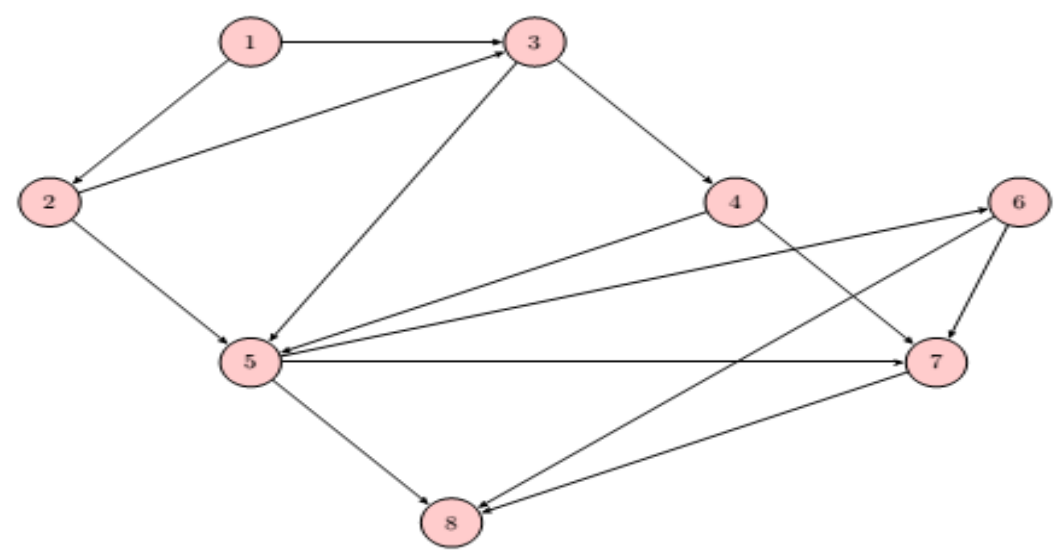

Figure 2 Network with Spherical fuzzy distance

The given network problem can be represented a as Spherical fuzzy digraph (SFDG), with 5 nodes as the service centers namely, 1, 2, 3, 4, 5, 6 and connection between the service center as the edges. The weights of each arc are given in Table 2 and for each node the labeling is given in Fig.2.

The following findings are obtained by utilizing the algorithm provided in section 4 .

Let the source node $(\mathrm{SN})=1$ and the terminus node $(\mathrm{TN})=8$, therefore $\mathrm{n}=8$.

Now, $\mathrm{p}_{1}=(0,0,1)$ and label $\mathrm{p}_{1}=[(0,0,1), 1]$ as the source node distance, and the Spherical fuzzy values of $p_{j}$, where $j=2,3,4,5,6,7,8$ can be attained from the following iterations: 
Iteration 0: Let us start with the source node $\mathrm{p}_{1}=(0,0,1)$ and label $\mathrm{p}_{1}=[(0,0,1), 1]$ as the source node distance, and the

Spherical fuzzy value of $p_{j}$, where $j=2,3,4,5,6,7,8$ can be obtained in the following iterations

Iteration 1: Node 2 has only one predecessor i.e., 1

$\mathrm{p}_{2}=\mathrm{p}_{1} \oplus \mathrm{p}_{12}=(0,0,1) \oplus(0.7,0.5,0.3)=(0.7,0.5,0.3)$ and label $\mathrm{p}_{2}=[(0.7,0.5,0.3), 1]$

Iteration 2: Node 3 has two predecessors namely, 1 and 2

$\mathrm{p}_{3}=\mathrm{p}_{1} \oplus \mathrm{p}_{13}=(0,0,1) \oplus(0.6,0.2,0.4)=(0.6,0.2,0.4)$ and $\mathrm{s}(0.6,0.2,0.4)=0.0(\mathrm{~min})$

$\mathrm{p}_{3}=\mathrm{p}_{2} \oplus \mathrm{p}_{23}=(0.7,0.5,0.3) \oplus(0.5,0.6,0.2)=(0.6175,0.52,0.06)$ and $\mathrm{s}(0.6175,0.52,0.06)=$

0.09920625

The label $\mathrm{p}_{3}=[(0.6,0.2,0.4), 1]$

Iteration 3: Node 4 has only one predecessor i.e., 3

$\mathrm{p}_{4}=\mathrm{p}_{3} \oplus \mathrm{p}_{34}=(0.6,0.2,0.4) \oplus(0.9,0.1,0.2)=(0.8784,0.0496,0.08)$

The label $\mathrm{p}_{4}=[(0.8784,0.0496,0.08), 3]$

Iteration 4: Node 5 has three predecessors namely, 2,3 and 4

$\mathrm{P}_{5}=\mathrm{p}_{2} \oplus \mathrm{p}_{25}=(0.7,0.5,0.3) \oplus(0.8,0.4,0.1)=(0.8164,0.37,0.03)$ and $\mathrm{s}(0.8164,0.37,0.03)=0.502825$

$\mathrm{P}_{5}=\mathrm{p}_{3} \oplus \mathrm{p}_{35}=(0.6,0.2,0.4) \oplus(0.7,0.5,0.4)=(0.6736,0.28,0.16)$ and $\mathrm{s}(0.6736,0.28,0.16)=0.249385$

(min)

$\mathrm{P}_{5}=\mathrm{p}_{4} \oplus \mathrm{p}_{45}=(0.8784,0.0496,0.08) \oplus(0.3,0.6,0.5)=(0.792144,0.361575,0.04)$ and

$\mathrm{s}(0.792144,0.361575,0.04)=0.46231$

The label $\mathrm{p}_{5}=[(0.6736,0.28,0.16), 3]$ 
Iteration 5: Node 6 has only one predecessor i.e., 5

$\mathrm{P}_{6}=\mathrm{p}_{5} \oplus \mathrm{p}_{56}=(0.6736,0.28,0.16) \oplus(0.6,0.2,0.1)=(0.650392,0.115264,0.016)$ and

$s(0.650392,0.115264,0.016)=0.392599$

The label $\mathrm{p}_{6}=[(0.650392,0.115264,0.016), 5]$

Iteration 6: Node 7 has three predecessors namely, 4, 5 and 6

$\mathrm{P}_{7}=\mathrm{p}_{4} \oplus \mathrm{p}_{47}=(0.8784,0.0496,0.08) \oplus(0.3,0.3,0.3)=(0.792144,0.092239,0.024)$ and

$\mathrm{s}(0.792144,0.092239,0.024)=0.585388$

$\mathrm{P}_{7}=\mathrm{p}_{5} \oplus \mathrm{p}_{57}=(0.6736,0.28,0.16) \oplus(0.5,0.2,0.2)=(0.590303,0.115264,0.032)$ and

$\mathrm{s}(0.590303,0.115264,0.032)=0.304769(\mathrm{~min})$

$\mathrm{P}_{7}=\mathrm{p}_{6} \oplus \mathrm{p}_{67}=(0.650392,0.115264,0.016) \oplus(0.6,0.1,0.1)=(0.630726,0.023153,0.0016)$ and $s(0.630726,0.023153,0.0016)=0.395335$

The label $\mathrm{p}_{7}=[(0.590303,0.115264,0.032), 5]$

Iteration 7: Node 8 has three predecessors namely, 5, 6 and 7

$\mathrm{P}_{8}=\mathrm{p}_{5} \oplus \mathrm{p}_{58}=(0.6736,0.28,0.16) \oplus(0.6,0.3,0.4)=(0.650392,0.161344,0.064)$ and

$s(0.650392,0.161344,0.064)=0.334379$

$\mathrm{P}_{8}=\mathrm{p}_{6} \oplus \mathrm{p}_{68}=(0.650392,0.115264,0.016) \oplus(0.8,0.3,0.1)=(0.792284,0.10209,0.0016)$ and

$s(0.792284,0.10209,0.0016)=0.615082$

$\mathrm{P}_{8}=\mathrm{p}_{7} \oplus \mathrm{p}_{78}=(0.590303,0.115264,0.032) \oplus(0.5,0.2,0.4)=(0.511343,0.052754,0.0128)$ and

$\mathrm{s}(0.511343,0.052754,0.0128)=0.246949(\mathrm{~min})$. The label $\mathrm{p}_{8}=[(0.511343,0.052754,0.0128), 7]$

By back tracing the minimum values of the above iterations, we get the Spherical fuzzy 
shortest path as $1 \rightarrow 3 \rightarrow 5 \rightarrow 7 \rightarrow 8$, with the Spherical fuzzy value $(0.511343,0.052754,0.0128)$ and the shortest distance is 0.246949 . Table 3 represents the SP from the source node to each $\mathrm{j}^{\text {th }}$ node and in Fig.4, the dark lines indicate the SP from the source node (service center) to the terminus node (customer).

Table 3 Shortest Spherical fuzzy path and their weights of each node from the source node.

\begin{tabular}{|l|l|l|}
\hline Node $\mathbf{j}$ & Shortest Spherical fuzzy Path from $\mathbf{1}$ to $\mathbf{j}$ & $\mathbf{p}_{\mathbf{i}}$ \\
\hline $\mathbf{2}$ & $1 \rightarrow 2$ & $(0.7,0.5,0.3)$ \\
\hline $\mathbf{3}$ & $1 \rightarrow 3$ & $(0.6,0.2,0.4)$ \\
\hline $\mathbf{4}$ & $1 \rightarrow 3 \rightarrow 4$ & $(0.8784,0.0496,0.08)$ \\
\hline $\mathbf{5}$ & $1 \rightarrow 3 \rightarrow 5$ & $(0.6736,0.28,0.16)$ \\
\hline $\mathbf{6}$ & $1 \rightarrow 3 \rightarrow 5 \rightarrow 6$ & $(0.650392,0.115264,0.016)$ \\
\hline $\mathbf{7}$ & $1 \rightarrow 3 \rightarrow 5 \rightarrow 7$ & $(0.590303,0.115264,0.032)$ \\
\hline $\mathbf{8}$ & $1 \rightarrow 3 \rightarrow 5 \rightarrow 7 \rightarrow 8$ & $(0.511343,0.052754,0.0128)$ \\
\hline
\end{tabular}

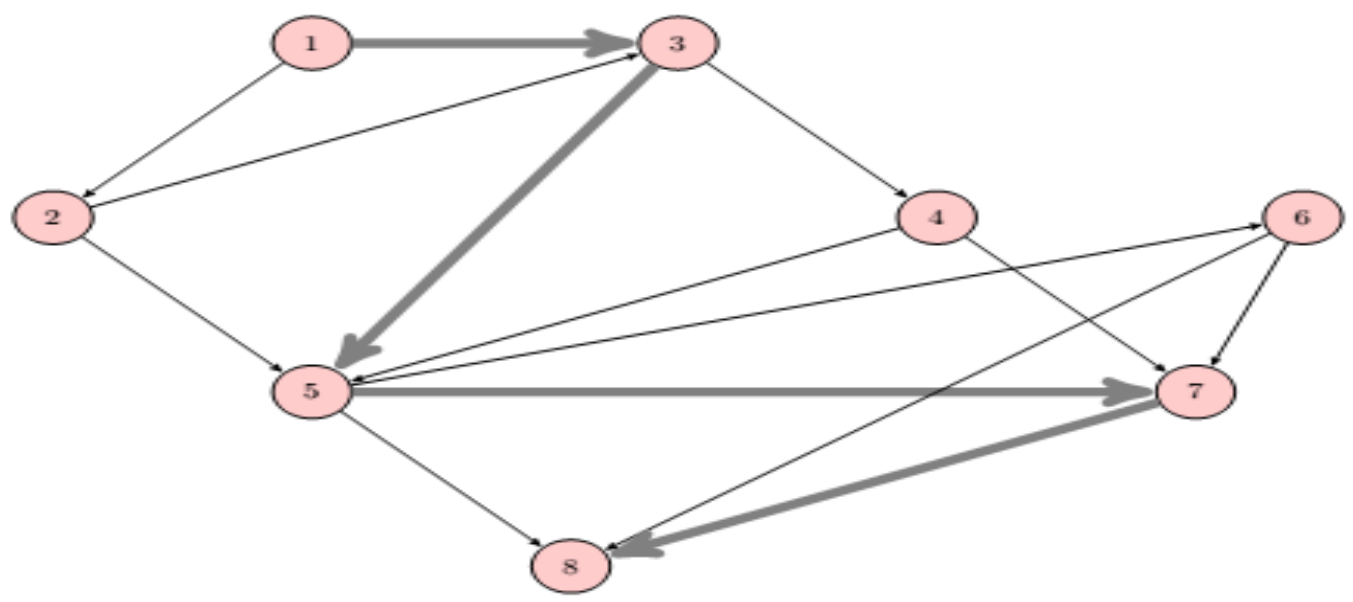

Figure 4 Network's Spherical fuzzy shortest path

\section{CONCLUSIONS}

The proposed algorithm is applied to solve the shortest path problem on a network with a Spherical fuzzy arc lengths in this paper. This algorithm is complete, optimal and finest one compared to other methods. This algorithm can be applied to solve a complex problem easily. 
Also this algorithm is optimally efficient, i.e. there is no other ideal algorithm that guarantees to give fewer nodes than the proposed one. A score function is used for defuzzification. We have enlightened the technique by a numerical example with the help of theoretical information. Furthermore, we can extend the proposed algorithm of the Spherical fuzzy shortest path problem into an interval valued Spherical fuzzy and bipolar Spherical fuzzy environment.

\section{REFERENCES}

Akram, M., Saleem, D. \& Hawary, T. A., (2020). Spherical Fuzzy Graphs with Application to Decision-Making, Math. Comput. Appl, 25(8): 1-32. doi:10.3390/mca25010008.

Ashraf, S., Abdullah, S., Aslam,M., Qiyas, M. \& Kutbi, M. A.,(2019). Spherical fuzzy sets and its representation of spherical fuzzy t-norms and t-conorms, Journal of Intelligent \& Fuzzy Systems 36: 6089-6102.

Atanassov, K., (1986). Intuitionistic fuzzy sets, Fuzzy Sets and Systems 20: 87-96.

Atanassov, K., (1988). Review and new results on Intuitionistic fuzzy sets, Preprint IMMFAIS-1-88, Sofia.

Atanassov, K. \& Stoeva, S.(1983). Intuitionistic fuzzy sets, in: Polish Syrup. on Interval \& Fuzzy Mathematics, Poznan, 23-26.

Broumi, S., Talea,M., Bakali,A., Smarandache,F., Nagarajan,D., Lathamaheswari,M. \&

Parimala,M.,(2019). Shortest path problem in fuzzy, intuitionistic fuzzy and neutrosophic environment: an overview, Complex \& intelligent Systems, Springer International Publishing: 1-8.

Cuong, B. C. \& Kreinovich, V.,(2013). Picture Fuzzy Sets-a new concept for computational intelligence problems, In Proceedings of the 2013 Third World Congress on Information and Communication Technologies (WICT2013), Hanoi, Vietnam 15(18): 1-6. 
Garg, H.,(2017). A novel improved accuracy function for interval valued Pythagorean fuzzy sets and its applications in the decision making process. Int J Intell Syst 32(12):1247-1260.

Garg, H.(2018a). Linguistic Pythagorean fuzzy sets and its applications in multi attribute decision-making process. Int J Intell Syst 33(6):1234-1263.

Garg, H.,(2018b). Hesitant Pythagorean fuzzy sets and their aggregation operators in multiple-attribute decision-making. Int J Uncertain Quantif 8(3):67-289.

Garg, H.,(2019). New logarithmic operational laws and their aggregation operators for Pythagorean fuzzy set and their applications. Int J Intell Syst 34(1):82-106.

Özdemir, Y.S. \& Üsküdar, A.(2020). Strategy selection by using interval type-2 fuzzy mcdm and an application Journal of Engg. Research. 8 (3): 172-189.

Parimala, M., Broumi, S. \& Karthika, M., (2019). A Network Shortest Path Algorithm via Hesitancy Fuzzy Digraph,Journal of New Theory, 27: 52-62.

Parvathi, R. \& Karunambigai, M.,(2006). Intuitionistic fuzzy graphs, In Computational Intelligence, Theory and Aplications, Springer: Berlin, Germany, 139-150.

Parvathi, R.\& Thamizhendhi, G., (2010). Domination in intuitionistic fuzzy graphs, Notes Intuit. Fuzzy Sets 16: 39-49.

Parvathi, R., Karunambigai, M. \& Atanassov, K. (2009). Operations on intuitionistic fuzzy graphs, In Proceedings of the 2009 IEEE International Conference on Fuzzy Systems, Jeju Island, Korea, 20-24

Rosenfeld, A.,(1975). Fuzzy Graphs, Fuzzy Sets and their Applications, Eds. Academic Press; New York, NY, USA.

Shannon, A. \& Atanassov, k.,(2006). On a generalization of intuitionistic fuzzy graphs. NIFS 12: 24-29.

Wei, G., Garg,H., Gao,H. \& Wei,C.(2018). Interval-valued Pythagorean fuzzy Maclaurin symmetric mean operators in multiple attribute decision making. IEEE Access 6:67866- 
67884.

Xu, Z. \& Hu, H.(2010). Projection models for intuitionistic fuzzy multiple attribute decision making, Int. J. Inf. Technol. Decis. Mak. 9: 267-280.

Yager, R. R. (2013). Pythagorean fuzzy subsets, Proceedings of the Joint IFSA World Congress and NAFIPS Annual Meeting, Edmonton, AB, Canada, 24-28.

Yager, R. R.(2014). Pythagorean membership grades in multi-criteria decision making. IEEE Trans. Fuzzy Syst. 22: 958-965.

Zadeh, L. A., (1965). Fuzzy Sets. Information and Control 18: 338-353. 